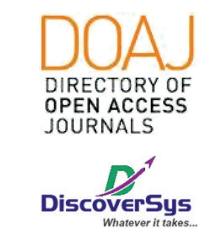

Published by DiscoverSys

\section{The correlation between location and size of infarction toward the quality of life in acute ischemic stroke patients at Sanglah General Hospital, Bali, Indonesia}

\author{
Mirani Ulfa Yusrika, ${ }^{1 *}$ Anak Agung Bagus Ngurah Nuartha, ${ }^{2}$ Ida Ayu Sri Wijayanti, ${ }^{2}$ \\ Made Widhi Asih, ${ }^{3}$ Skolastika Savitri Sujatmiko
}

\section{ABSTRACT}

\begin{abstract}
Background: Location and size of infarction in acute ischemic stroke can cause different levels of neurological deficits and disabilities according to the function of the area of brain whose blood flow is disrupted, thus affecting the quality of life in patients. This study was aimed to explore the correlation between location and size of infarction toward the quality of life in acute ischemic stroke patients in Sanglah General Hospital Denpasar.

Methods: A cross-sectional study was conducted among 41 acute ischemic stroke patients at Neurology Care Unit, Sanglah General Hospital Denpasar during May-September 2019. The location of the infarct was grouped by the cerebral area. The largest diameter of infarction determined the size of the infarct. Data were taken from head Computed Tomography (CT) scan results to assess the location and size of infarction and Barthel Index (BI) to measure the quality of life-based on Activity
\end{abstract}

of Daily Living (ADL). Data were analyzed using univariate and bivariate analysis (Chi-Square test with Cramer's V for location and BI scores, Spearman test for size and BI scores) on SPSS version 23 for Windows. Results: Most of the respondents were males 59.0\%, the average age of $60.03 \pm 14.4$ years, ischemic thrombus stroke $(71.8 \%)$, subcortical infarction (41.0\%), small size $(\leq 1.5 \mathrm{~cm})$ of infarction $(48.7 \%)$, and independent criteria for Barthel Index Scores (23.1\%). There were significant correlations between the location of infarction and $\mathrm{BI}$ scores $(r=0.636 ; p<0.000)$ and the size of infarction and $B I$ scores $(r=0.553$; $p<0.000$ ).

Conclusion: Infarct that located in the cortical area with small size are related to independent or mild dependency outcome. Conversely, infarct that located and associated with a subcortical area with larger size are related to severe or total dependency.

Keywords: Ischemic Stroke, Infarct Location, Infarct Size, Barthel Index Scores.

Cite This Article: Yusrika, M.U., Nuartha, A.A.B.N., Wijayanti, I.A.S., Asih, M.W., Sujatmiko, S.S. 2020. The correlation between location and size of infarction toward the quality of life in acute ischemic stroke patients at Sanglah General Hospital, Bali, Indonesia. Intisari Sains Medis 11(2): 717-721. D0I: 10.15562/ism.v11i2.675

'Undergraduate Student, Faculty of Medicine, Universitas Udayana, Bali, Indonesia

${ }^{2}$ Department of Neurology, Faculty of Medicine, Universitas Udayana, Sanglah General Hospital, Bali, Indonesia

${ }^{3}$ Department of Radiology, Faculty of Medicine, Universitas Udayana, Sanglah General Hospital, Bali, Indonesia

\section{*Correspondence to:}

Mirani Ulfa Yusrika; Undergraduate Student, Faculty of Medicine, Universitas Udayana, Bali, Indonesia; miraniulfay@gmail.com

Received: 2019-12-16 Accepted: 2020-06-28 Published: 2020-08-01

\section{INTRODUCTION}

Stroke is the leading cause of disability in the world. As many as $80 \%$ of total stroke cases are ischemic strokes. ${ }^{1}$ Ischemic stroke occurs when the blood supply to the brain is disrupted due to occlusion that can be caused by thrombus or embolism or both. ${ }^{2}$ The hypoperfusion will be altered the function of the brain, resulting in neurological deficits and disability. ${ }^{1-3}$ This phenomenon causes limitations in patients to perform necessary activities of daily living (ADL). ${ }^{4}$ The stroke patients experience changes in living their lives, such as changes in daily activities, social relationships, communication, and psychological conditions, both inpatients and in their families. ${ }^{4,5}$ These aspects are an indicator that shows an alteration in the quality of life of stroke patients. ${ }^{3-5}$

The damage to the brain causes different levels of neurological deficits and disability according to the function of the part of the brain whose blood flow is disrupted. ${ }^{5}$ Thus it can be a predictor of the functional outcome..$^{3-5}$ Neuroimage findings of the brain damage, such as the location and size of infarction, can be evaluated and may be correlated with the ADL outcome that can be an indicator of the quality of life, through Barthel Index (BI) score in patients with acute ischemic stroke.,7

Based on those mentioned above, this study aims to evaluate the correlation between location and size of infarction toward the quality of life in acute ischemic stroke patients at Sanglah General Hospital, Bali, Indonesia.

\section{METHODS}

\section{Inclusion and Exclusion Criteria}

This study was a cross-sectional observational analytic study with a consecutive non-random sampling method conducted during May September 2019 at Sanglah General Hospital, 
Denpasar, Bali, Indonesia. The targeted sample included all acute ischemic stroke patients who were admitted to the Neurology Care Unit during the time of the study that met these criteria: (1) Diagnosed with acute ischemic stroke with onset $<72$ hours for the first time, (2) The patients' age over 30 years old, and (3) The ischemic stroke confirmed by head CT scan with cerebral or supratentorial infarction findings. Acute ischemic stroke patients who were not confirmed by head CT scan or with other infarction findings such as in pons, cerebellum, or medulla oblongata were excluded due to different vascularization. Patients with the second attack of ischemic stroke, hemorrhagic stroke, other brain abnormalities, pregnancy, and impaired immunity, were also excluded since it can affect the BI scores due to severe impairments.

In brief, 41 patients who had the first time, acute ischemic stroke were recruited. Among 41 patients, 39 patients met the inclusive criteria. The others were excluded because one patient was pregnant, and one patient with ischemic infarction in the pons.

\section{Data Collection}

The data were collected from head CT scan results to assess the location and size of infarction and from BI scores to measure the quality of life-based on ADL. The head CT scan was administrated to the patient on the first day of admission as the gold standard to diagnose ischemic stroke. The location of infarction was grouped by the cerebral area, including cortical area, subcortical area, and cortical-subcortical area. The size of infarction was determined by the largest diameter of infarction in centimeters and later grouped into 3 categories, small $(\leq 1.5 \mathrm{~cm})$, medium $(>1.5-<5 \mathrm{~cm})$, and large $(\geq 5 \mathrm{~cm})$.

BI scores consist of 10 points for assessing the ability of the patient to do self-care, mobility, or ADL, including the patients' ability in feeding, bathing, personal hygiene, dressing, bowel and bladder care, transfer, ambulation, and stairclimbing. The interpretation of BI scores will be classified into 5 categories, independent (80-100), mild dependency (60-79), moderate dependency (40-59), severe dependency (20-39), and total dependency (0-20). The BI scores from each patient were assessed on the last day or the $7^{\text {th }}$ day of admission in Neurology Care Unit. ${ }^{5,6}$

Other factors that can affect the quality of life of the patients, such as age, gender, and types of ischemic stroke, are also obtained and will be analyzed and displayed on the baseline characteristic of acute ischemic stroke patients. All the data was written down to the data collection sheet.

\section{Data Analysis}

The data were analyzed by Statistical Package for Social Sciences (SPSS) program version 23. The data will be in descriptive analysis for the baseline characteristic of acute ischemic stroke patients and analytical analysis to discover any correlation. The location of infarction and BI scores were analyzed using Chi-Square Test with Cramer's V, while the size of infarction and BI scores were analyzed using Spearman Test. Chi-Square Tests are usually used to analyze the correlation between two categorical variables (nominal and either nominal or ordinal variables), whether it is statistically significant or not. The location of infarction is a nominal variable while the BI scores is an ordinal variable. Cramer's $\mathrm{V}$ is a part of the Chi-Square Test that performed to measure the strength of the correlation. While Spearman Test is usually used to analyze the correlation between two ordinal variables, both sizes of infarction and BI scores are ordinal variables.

\section{RESULTS}

The average age of the patients was $60.03 \pm 14.4$ years old. Among the samples, more than half of the patients were male $(59.0 \%)$, and $71.8 \%$ had thrombus type of ischemic stroke (Table 1). In addition, most infarcts were located in the subcortical area (41.0\%), while the size of most infarcts was a small size (48.7\%) (Table 1). The Barthel Index (BI) scores of the patients were predominant in the independent (80-100) criteria (23.1\%) (Table 1).

According to the Chi-Square with Crammer's V test, the recent study found a significant correlation between the location of infarction and BI scores $(r=0.636 ; p<0.000)$ that presented in Table 2. In addition, based on the Spearman correlation test, there was a strong significant positive correlation between the size of infarction and BI scores $(\mathrm{r}=0.553 ; \mathrm{p}<0.000)$ in Table 2.

\section{DISCUSSION}

The results of this study indicate that there was a significant correlation between the location of infarction and BI scores $(\mathrm{p}<0.000)$. However, the correlation coefficient between the location of infarction and BI scores was 0.636 , indicating a strong correlation. The higher score of correlation coefficient, the more strongly the variables correlated, and one variable can easily determine the other. Among various infarct locations, the subcortical area showed the most favorable outcome with lesser improvement in BI scores compared to the cortical area. This result indicated that infarction located in the cortical area is related to independent 
Table 1 Baseline Characteristic of Samples

\begin{tabular}{|c|c|c|c|}
\hline Variable & Participants $(\mathrm{N}=39)$ & Percentage (\%) & Mean \pm SD \\
\hline \multicolumn{4}{|l|}{ Age (Years) } \\
\hline$\leq 50$ years & 10 & 25.6 & \\
\hline$>50$ years & 29 & 74.4 & \\
\hline \multicolumn{4}{|l|}{ Gender } \\
\hline Male & 23 & 59.0 & \\
\hline Female & 16 & 41.0 & \\
\hline \multicolumn{4}{|l|}{ Type of Ischemic Stroke } \\
\hline Thrombus & 28 & 71.8 & \\
\hline Embolism & 11 & 28.2 & \\
\hline \multicolumn{4}{|l|}{ Location of Infarction } \\
\hline Cortical & 12 & 30.8 & \\
\hline Subcortical & 16 & 41.0 & $60.03 \pm 14.4$ \\
\hline Cortical - subcortical & 11 & 28.2 & \\
\hline \multicolumn{4}{|l|}{ Size of Infarction } \\
\hline Small $(\leq 1.5 \mathrm{~cm})$ & 19 & 48.7 & \\
\hline $\operatorname{Medium}(>1.5-<5 \mathrm{~cm})$ & 13 & 33.3 & \\
\hline Large $(\geq 5 \mathrm{~cm})$ & 7 & 18.0 & \\
\hline \multicolumn{4}{|l|}{ Barthel Index (BI) Scores } \\
\hline Independent $(80-100)$ & 9 & 23.1 & \\
\hline Mild dependency $(60-79)$ & 8 & 20.5 & \\
\hline Moderate dependency $(40-59)$ & 8 & 20.5 & \\
\hline Severe dependency $(20-39)$ & 8 & 20.5 & \\
\hline Total dependency (0-20) & 6 & 15.4 & \\
\hline
\end{tabular}

Table 2 Chi-Square Test with Cramer's V between Location of Infarction and Barthel Index Scores

\begin{tabular}{lccccccc}
\hline & \multicolumn{5}{c}{ Barthel Index Scores } & & \\
\cline { 2 - 5 } Variables & Independent & Mild & Moderate & Severe & Total & & r \\
\hline Location & 7 & 5 & 0 & 0 & 0 & $0.636^{\mathrm{a}}$ & $<0.000^{\mathrm{a}^{*}}$ \\
$\quad$ Cortical & 0 & 3 & 7 & 4 & 2 & & \\
$\quad$ Subcortical & 2 & 0 & 1 & 4 & 4 & & \\
$\quad$ Cortical-Subcortical & & & & & & & \\
Size & 7 & 6 & 4 & 2 & 0 & $0.553^{\mathrm{b}}$ & $<0.000^{\mathrm{b}^{*}}$ \\
$\quad$ Small & 1 & 2 & 3 & 4 & 3 & & \\
$\quad$ Medium & 1 & 0 & 1 & 2 & 3 & \\
$\quad$ Large & & & & & & & \\
\hline
\end{tabular}

Statistically significant if $\mathrm{p} \leq 0.05$

${ }^{\mathrm{a}} \mathrm{Chi}-\mathrm{Square}$ and Cramer's V used to measure the strength of the correlation: $0.00-0.15=$ very weak; $0.15-0.20=$ weak; $0.20-0.25=$ moderate; $0.25-0.30=$ moderately strong; $0.30-0.35=$ strong; $0.35-0.40=$ very strong; $0.40-0.50=$ worrisomely strong; $0.50-0.99=$ redundant; $1.00=$ perfect relationship.

${ }^{\mathrm{b}}$ Spearman Correlation test between size of infarction and BI scores; $\mathrm{r}=$ correlation coefficient. The interpretations: $0.00-.19=\mathrm{very}$ weak; $0.20-0.39=$ weak; $0.40-0.59=$ moderate; $0.60-0.79=$ strong; $0.80-1.0=$ very strong.

or mild dependency outcomes. In contrast, the infarction that is related to the subcortical area, the outcome refers to severe or total dependency.

This finding has a similarity to the previous study by Pan SL et al., which showed a significant relationship between infarct location and BI scores. ${ }^{4}$ The study revealed that the location of infarction in the cortical area was correlated with higher BI scores, which means that the patients have a better quality of life with more independent ADL, and 
vice versa. ${ }^{4}$ It was similar to the study by Yong SW et al., and Dhamoon et al., stated that significantly more patients with the infarct location involving the subcortical area had a poor outcome. ${ }^{8,9}$ A study by Hussain $\mathrm{Z}$ et al. also revealed similar research results; there was a significant correlation between infarct locations and BI scores $(\mathrm{p}=0.004) .^{7}$ The study explained, based on ADL outcomes in patients with severe dependence, the infarcts in the corticalsubcortical area as many as 22 of 28 patients $(78.6 \%)$, in the subcortical area as many as 29 of 67 patients (43.3\%), and in the cortical area 3 out of 8 patients (37.5\%). In addition, a study conducted by Nazzal et al. found that the highest specific proportion based on ADL levels with higher or more independent values was found in patients with infarction in the cortical area (61.5\%). ${ }^{10}$

In our study, the size of infarction had a significant correlation on BI scores with the results of this study. The strength of the correlation between the size of infarction and BI scores was 0.553 , indicating a moderate correlation. This result showed that the larger the infarct size, the functional outcome will be more dependent and less favorable, and the smaller the size of the infarction, the higher the IB value, which means that the ADL outcome of ischemic stroke patients is more independent.

Previous research by Pan SL et al. had a similar result, the correlation between the size of infarction based on the longest diameter and BI scores also showed significant results $(\mathrm{p}=0.0001) .{ }^{4}$ This study suggests that patients with small infarcts $(\leq 1.5 \mathrm{~cm})$ had prompt recovery early. ${ }^{4}$ Another study by Kalowska E et al. also had a similar result, stated that the larger the size of the infarction, the lower the BI scores, which means that the ADL outcome of ischemic stroke patients is more dependent. ${ }^{11}$ However, a study by Barrett et al. show different results. ${ }^{12}$ This study used two methods to measure the functional outcome of ischemic stroke, BI scores, and modified Rankin Scale (mRS). ${ }^{12}$ The results of this study revealed that there was no significant correlation between infarct size and BI scores $(\mathrm{p}=0.056)$. However, when using mRS, there is a considerable correlation between infarct size and mRS scores $(p=0.0001)$. It can be concluded that there is a correlation between the size of infarction and functional outcomes of acute ischemic stroke patients but by using a different measurement method, mRS. ${ }^{12}$

The major finding of this study was both location and size of the infarction were associated with BI scores. The location and size of the infarction have been shown to play a part in defining the quality of life of patients with acute ischemic stroke. Several previous studies showed that the infarct location involving the subcortical area has a less favorable functional outcome. ${ }^{10-12}$ The reason for this can be explained; there are a lot of major sensorymotoric pathways assembled in this location. If brain injuries like ischemic stroke are involved in this location, the patients more likely to have severe neurological deficit. ${ }^{7-10}$ The size of the infarction was significantly correlated with BI scores. ${ }^{7-10}$ Several previous studies revealed that the larger the infarct size suggests bigger neural impairment, lead to the greater neurological deficit and poorer outcome. ${ }^{11-13}$

\section{CONCLUSION}

Based on the results of this study and supporting data from previous studies, it shows that the quality of life of acute ischemic stroke patients based on the ADL level of BI scores in the Neurology Care Unit of Sanglah Hospital Denpasar is associated with the location and size of the infarction. For further research, it is advisable further to investigate the infarct location in the infratentorial area, measure the infarct size by other measurement methods, and use other functional outcome measurement methods. Larger sample size is recommended to resolve the limitation of this study.

\section{ETHICAL CLEARANCE}

This study has been obtained ethics approval from The Ethics Committee, Faculty of Medicine, Udayana University/Sanglah General Hospital, Denpasar, Bali, Indonesia with the references number LB.02.01/XIV.2.2.1/13451/2018

\section{CONFLICT OF INTEREST}

The authors declare that there isino competing interest regarding the publication of this article.

\section{FUNDING}

The authorsbare responsible for the funding of the study without the involvement of a grant, scholarship, or any other resources of funding.

\section{AUTHOR CONTRIBUTIONS}

All authors are equally contributed to this research from conceptual framework, data gathering, data analysis until results interpretation in the final report. 


\section{REFERENCE}

1. Sacco RL, Kasner SE, Broderick JP, Caplan LR, Connors JJB, Culebras A, et al. An updated definition of stroke for the 21st century: a statement for healthcare professionals from the American Heart Association/American Stroke Association. Stroke. 2013;44(7):2064-2089.

2. Kanyal N. The Science of Ischemic Stroke: Pathophysiology \& Pharmacological Treatment. IJPRR. 2015;4(10):65-84.

3. Caplan LR, Wong KS, Gao S, Hennerici MG. Is hypoperfusion an important cause of strokes? If so, how?. Cerebrovasc Dis. 2006;21(3):145-153.

4. Pan SL, Wu SC, Wu TH, Lee TK, Chen TH. Location and size of infarct on functional outcome of noncardioembolic ischemic stroke. Disabil Rehabil. 2006;28(16):977-983.

5. López-EspuelaF,Pedrera-ZamoranoJD,Jiménez-CaballeroPE, Ramirez-Moreno JM, Portilla-Cuenca JC, Lavado-Garcia JM, et al. Functional Status and Disability in Patients After Acute Stroke: A Longitudinal Study. Am J Crit Care. 2016;25(2):144-151.

6. Quinn TJ, Langhorne P, Stott DJ. Barthel index for stroke trials: development, properties, and application. Stroke. 2011;42(4):1146-1151

7. Hussain Z, Hilal K, Ahmad M, Sajjad Z, Sayani R. Clinicoradiological Correlation of Infarct Patterns on Diffusion-weighted Magnetic Resonance Imaging in Stroke. Cureus. 2018;10(3):e2260.

8. Yong SW, Bang OY, Lee PH, Li WY. Internal and cortical border-zone infarction: clinical and diffusion-weighted imaging features. Stroke. 2006;37(3):841-846.
9. Dhamoon MS, Cheung YK, DeRosa JT, Gutierrez J, Moon YP, Sacco RL, et al. Association Between Subclinical Brain Infarcts and Functional Decline Trajectories. J Am Geriatr Soc. 2018;66(11):2144-2150

10. Nazzal ME, Saadah MA, Saadah LM, Trebinjac SM. Acute ischemic stroke: relationship of brain lesion location \& functional outcome. Disabil Rehabil. 2009;31(18):1501-1506.

11. Kalowska E, Rostrup E, Rosenbaum S, Petersen P, Paulson OB. Acute MRI changes in progressive ischemic stroke. Eur Neurol. 2008;59(5):229-236.

12. Barrett KM, Ding YH, Wagner DP, Kallmes DF, Johnston KC; ASAP Investigators. Change in diffusionweighted imaging infarct volume predicts neurologic outcome at 90 days: results of the Acute Stroke Accurate Prediction (ASAP) trial serial imaging substudy. Stroke. 2009;40(7):2422-2427.

13. Yuantari R, Wuriyanti D, Siswantoro D. The correlation of Lipoprotein (a) with the severity of ischemic stroke in Dr. Soedono General Hospital, Madiun, Indonesia. Bali Medical Journal. 2019;8(1):275-280.

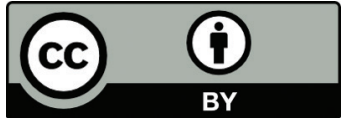

This work is licensed under a Creative Commons Attribution 